\title{
Meeting Report \\ Replacing Fetal Bovine Serum (FBS) in Research and Testing
}

doi:10.14573/altex.2012141

Fetal bovine serum (FBS) is used by default as an additive for cell, organ and tissue culture media in biomedical research and testing to enhance cell growth and proliferation. These cultures are valuable as a means of replacing and reducing animal experiments. However, despite this potential, there are severe animal welfare issues associated with the collection of FBS, since it is derived from animal sources and its production and use are associated with ethically and legally questionable practices, scientific challenges, and animal suffering.

It is, therefore, of paramount importance to draw the attention of the scientific community towards this topic to increase the awareness and acceptance of alternatives to FBS and to train scientists and lab technicians in transition techniques to FBS-free culture media to contribute to the development of truly animal-free alternatives to animal experiments.

Thanks to the generous financial support of the Renate Benthlin-Stiftung für Nutztierschutz, the Animal Welfare Academy of the German Animal Welfare Federation was able to organize a fullday online symposium on replacing FBS. Speakers from universities, industry, regulatory authorities, and animal welfare NGOs presented the current status of the collection and use of FBS, and the ethical and scientific advantages of FBS-free alternatives.

Tilo Weber, scientific officer at the Animal Welfare Academy of the German Animal Welfare Federation reported on the massive animal suffering in the production of FBS, which is harvested from the blood of bovine fetuses. Distress and suffering already begin with the transport of pregnant dams to the slaughterhouse. Therefore, veterinary examinations should be implemented beforehand to avoid transportation and slaughter of pregnant animals. Furthermore, the calf fetuses are neither killed by a certified humane killing method that fulfills animal welfare standards nor do they receive pain relief or anesthetics during the process. As long as FBS is still produced, legally binding regulations are indispensable that must at least implement mandatory anesthesia and/or humane killing of the fetuses.

Dr Jan van der Valk, Director of the 3Rs-Centre Utrecht Life Sciences, pointed out the scientific challenges of using FBS: The exact composition of FBS is unknown, there is a risk of contamination with pathogens, and reproducibility of scientific experiments suffers from inconsistencies between FBS batches. Therefore, xenofree media (e.g., human-based medium for human cells) or fully chemically-defined media are key to ensure scientific reproducibility. He gave insight into the establishment of the database for FBS-free media ${ }^{1}$ hosted by the 3 Rs-Centre Utrecht Life
Sciences, which he initiated. He encouraged scientists to use the database and contribute their own formulations and experience with alternative media.

Subsequently, a human-based supplement medium was presented, which consists of human platelet lysates (hPL) obtained from expired blood donations. Dr Karen Bieback, professor at the University of Heidelberg and head of the stem cell quality control lab at the German Red Cross Blood Donor Service Institute Mannheim, highlighted the advantages of this medium for use in stem cell cultures.

The regulatory perspective was reflected by Dr Sandra Coecke from the Joint Research Center of the European Commission (JRC, EURL-ECVAM). She talked about the advantages of chemically-defined media and explained her work on the Guidance Document on Good In Vitro Method Practices (GIVIMP), a key tool for ensuring reproducible study data generation omitting ill-defined animal-derived ingredients like FBS.

Two practical applications for cell cultures in FBS-free media were presented next. Dr Aline Chary from the Luxembourg Institute for Science and Technology (LIST) used the alveolar epithelial type II A549 cell line as an example to explore how to transition cells to FBS-free media. She works towards the goal of complete replacement of animal-derived components for cell culture of A549 cells, which are commonly used in respiratory toxicology testing.

Dr Joachim Wiest (CEO of cellasys $\mathrm{GmbH}$ ) presented the cellasys \#8 assay, a protocol to investigate the interaction between cell culture medium and cellular models by means of extracellular acidification rate (EAR) measurement and electric cell-substrate impedance sensing (ECIS). Using this assay, the suitability of FBSfree media for cell cultures can be assessed within $24 \mathrm{~h}$.

A wide variety of topics were discussed during the panel discussion, including positive and negative experiences with FBSfree media, proprietary media formulations, and comparisons between the costs of FBS and alternative media. It was emphasized that even though alternatives to FBS might still be more costly, supposedly lower prices for a medium supplement that is ethically questionable, unregulated, and undefined can have far greater negative financial effects on research work due to potential scientific inaccuracies and lack of reproducibility.

A controversial topic was the state of the fetus during blood extraction. If blood is collected from a living fetus in the last third of its development, it should be regarded as a procedure according to Directive 2010/63/EU. However, even if the animal dies before

\footnotetext{
1 https://fcs-free.org/
} 
blood extraction begins, animal welfare issues remain, as potential suffering is not alleviated by anesthetizing or euthanatizing the fetus, and the animals are simply left to asphyxiate. Thus, a binding regulatory control of FBS production must be implemented that takes animal welfare and ethical concerns into account.

After the panel discussion, Dr Wiest presented a video to demonstrate the application protocol of the cellasys \#8 assay.

Based on the positive feedback on the presentations and the panel discussion, the consensus of the more than 50 participants from 13 countries was that FBS is not a suitable and future-proof medium supplement for an exact and humane science and that only a joint effort by industry, authorities, universities and animal welfare can contribute to a paradigm shift towards more reliable research and testing methods. One of the next steps is to contact the serum-producing industry to encourage discussions to find a common solution for the animal welfare issues and the development of animal-friendly media supplements.

A detailed report on the symposium is in progress as well as a joint publication by the speakers on alternatives to FBS. A handson workshop in cooperation with cellasys $\mathrm{GmbH}$ (again with the support of the Renate-Benthlin Stiftung für Nutztierschutz) will be held in 2021. Therein, participants will be trained on how to perform the cellasys \#8 assay to support the transition to FBS-free media for the cell and tissue cultures they are using.

\section{Tilo Weber and Kristina Wagner}

Animal Welfare Academy of the German Animal Welfare Federation, Neubiberg, Germany

\title{
Meeting Report
}

\section{A New Gold Standard: Paradigm Shift in Research on Human Diseases and Therapy Developments - Animalfree Research Forum 2020}

\author{
doi:10.14573/altex.2011301
}

On November 19, 2020, the Swiss-based foundation Animalfree Research held its $10^{\text {th }}$ forum. This year's topic was "A new gold standard: paradigm shift in research on human diseases and therapy developments". The forum was held online (Fig. 1) and attracted over 60 participants from both Switzerland and abroad.

Dr Silvia Frey, Director at Animalfree Research, opened the forum, introducing the goals of Animalfree Research and the aims of the forum.

Dr Miriam Zemanova, Scientific Associate at Animalfree Research, kicked the meeting off with an introductory talk on the topic. Dr Zemanova spoke about the high failure rate in drug development due to the low predictive power of animal testing in biomedical research, the reasons why animals constitute poor models of human diseases, and what the barriers to achieving the shift from animal testing to human-relevant, animal-free methods might be.

Dr Martin Smieško, Research Associate at the Department of Pharmaceutical Sciences, University of Basel, spoke on "In silico screening methods for 3R: theory and practice". In silico methods can be employed to screen enormous amounts of data to find the best possible chemical compound for interaction with a specific biological target, yielding a desired pharmacological effect. Compound toxicity can be evaluated either through similarity search or through creating a computer model for the observed phenomenon. Dr Smieško's group has been pioneering the use of in silico screening techniques, implemented in the VirtualToxLab platform, for early recognition of the off-target binding of drugs, chemicals, and natural compounds, with the ambition to reduce the extent of animal testing. Dr Smieško explained that the pharmaceutical field has relied too much on animal studies and that animal testing is being implemented too early in drug development. He concluded that in silico technology has great potential that is yet to be unleashed in toxicology and pharmacology.

Dr Samuel Constant, CEO of Epithelix, held a lecture entitled "3D human airway epithelial models to study SARS-CoV-2 pathogenesis". Dr Constant provided an overview of SARSCoV-2 infection, which affects the airway epithelial cells. Epithelix has developed standardized air-liquid interface 3D human airway epithelial cultures of nasal or bronchial (MucilAir ${ }^{\mathrm{TM}}$ ) and small-airway (SmallAir ${ }^{\mathrm{TM}}$ ) origins. These models closely mimic the morphology and function of the native tissues: cilia formation and beating, mucus production and secretion, mucociliary clearance, and secretion of antiviral molecules. Epithelix have successfully used their innovation for the development of antivirals against influenza, rhinoviruses, respiratory syncytial virus, and others. These reconstituted human airway epithelial models can be used to characterize viral infection kinetics, tissue-lev- 